\title{
Results of surgery using a locking plate for proximal humerus fractures in patients older than 80 years: $A$ retrospective case series
}

Won Chul Shin

Pusan National University Yangsan Hospital

Suk-Woong Kang ( $\nabla$ redmaniak@naver.com )

Pusan National University Yangsan Hospital https://orcid.org/0000-0003-0883-2461

Seung Min Son

Pusan National University Yangsan Hospital

Hyuk Bae

Daedong Hospital

Research article

Keywords: elderly patients, proximal humeral fracture, locking plate fixation

Posted Date: November 5th, 2019

DOI: https://doi.org/10.21203/rs.2.14214/v2

License: (c) (i) This work is licensed under a Creative Commons Attribution 4.0 International License.

Read Full License 


\section{Abstract}

Background: To evaluate the results of surgical treatment using a locking plate for proximal humeral fractures in patients aged $>80$ years. Methods: Between September of 2013 and March of 2016, there were 22 patients who received locking plate fixation from proximal humeral fractures over 80 years-old. Among the 22 cases, Clinical, radiological results were analyzed for 19 patients who were able to follow up more than one year. We analyzed bone union, neck-shaft angle, UCLA score, range of motion compared to opposite side and complication. Clinical, radiological results were investigated for medial comminuted fracture or not. Results: All the patients achieved bone union. The mean bone union time was 13.7 weeks, and the mean neck-shaft angle was 126.4. The mean University of California, Los Angeles, shoulder score was 22.4 , and score was $<28$ point in 12 patients. The mean forward flexion, abduction, external rotation, and internal rotation angles were $129.2^{\circ}, 112.3^{\circ}, 44.2^{\circ}$, and L2. All motions were significantly different from the normal shoulder motion. A significant difference was found in the loss of neck-shaft angle according to the medial comminuted fracture. Conclusion: In the surgical treatment of proximal humeral fractures in patients aged $>80$ years, use of a locking plate attained bone union with relatively satisfactory results. However, we considered that prevention of and training for postoperative stiffness are necessary. Other surgical methods should be considered for patients with complex displaced fractures, especially those with medial comminuted fractures.

\section{Background}

Proximal humeral fracture is the third most common fracture and occurs in approximately $6 \%$ of total fractures, with increasing incidence recently [21]. Conservative treatment is performed in older patients with low expectations due to medical problems, and good results can be expected in the absence of displacement or in simple two-part fractures $[12,15]$. However, in patients with displaced, comminuted, or angulated fractures proximal humeral fractures, surgical treatment should be performed to restore shoulder function with firm fixation and early mobilization. In these cases we can perform several methods of surgery, percutaneous techniques, intramedullary nailing, plating, or arthroplasty.[3, 27] Some authors recommend percutaneous pinning or screw fixation for 2-part fractures in the elderly patient.[5] [27]Intramedullary nails have the benefit of limited soft tissue dissection and blood loss for 2-par fracture, but there many complications like distraction, screw back-out and nonunion.[3] Open reduction and locking plate fixation is generally chosen $[16,24]$. However, in elderly patients, the frequency of complications such as varus deformity and screw penetration due to osteoporosis is high. Especially in combined medial comminuted fractures, loss of fracture reduction occurs more frequently $[17,20]$. Therefore, the number of reverse total shoulder arthroplasty cases is increasing in elderly patients [16]. Some authors said that the preference for RSA over HA was especially high among patients aged 80 years or older, because they have a higher prevalence rotator cuff disease. $[13,30]$ Furthermore, Patients over 80 years of age are considered a high risk group of fractures.[2, 8]

There are reports of surgical outcomes in older patients with proximal humeral fractures, but very few reports have been reported on locking plates in very elderly patients. In this regard, we would like to know 
how poor surgical results in these patients is and how many complications they have. Therefore, the purpose of this study was to analyze the results of surgical treatment using a locking plate for proximal humeral fractures in patients aged $>80$ years. Furthermore, we examined the radiological and clinical differences among elderly patients with medial comminuted fractures and complications of plate fixation for proximal humeral fractures.

\section{Methods}

This was a retrospective study, and final approval of informed consent exemption by the institutional review board was obtained (IRB NO. 05-2019-013).

Patients who underwent locking plate fixation for the treatment of proximal humeral fracture by the single author at one institution between September 2013 and March of 2016 were included. We performed reverse total shoulder arthroplasty for proximal humeral fractures in patients aged $>80$ years who had cuff arthropathy or glenohumeral arthritis as diagnosed by radiography and computed tomography (CT). Twenty-two patients aged $>80$ years received locking plate fixation for proximal humeral fractures. Among the 22 patients, 3 were excluded, including 2 who died within 1 year after surgery for unrelated causes and one who had a follow-up period of only 8 months. Finally, we retrospectively evaluated 19 patients ( 4 men and 15 women) who underwent locking plate fixation with proximal humeral fractures within the same period and were followed up for at least 1 year. The mean age at the time of surgery was $82.2 \pm 3.08$ years (range, $80-91$ years), and the mean follow-up duration was $15.4 \pm 5.09$ months (range, 12-39 months). By using dual-energy X-ray absorptiometry, we measured the bone density of the patients' hip and spine, which according to the lowest T-score, showed a mean bone mineral density of $-2.8 \pm 0.86$ (range, -1.3 to -5.9 ). According to the Neer classification system, 9 patients had two-part fractures, 8 had three-part fractures, and 2 had four-part fractures (Table 1).

Radiological evaluation was performed immediately and at 2 weeks, 4 weeks, 3 months, 6 months, 9 months, and 12 months after surgery. We defined successful bone union when the fracture site was completely adjoined through callus formation. Humeral neck-shaft angles (NSAs) were measured in the anteroposterior plane. We defined humeral NSA as the angle formed between the line perpendicular to the articular surface and the line following the humeral shaft. The humeral NSA was measured preoperatively, postoperatively, and at the final follow-up. (Figure 1.) Clinical results were compared with University of California Los Angeles (UCLA) shoulder score,[1] and range of motion was compared with the that of the opposite arm. The results in relation to the medial comminuted fracture and other complications were analyzed.

We used SPSS Statistics ver. 17.0 (SPSS Inc., Chicago, IL, USA) to perform all statistical analyses. The nonparametric Mann-Whitney $U$ test was used to analyze the data. The significance threshold was set at 0.05 .

\section{Results}


We achieved successful bone union in all the patients, and the mean time to bone union was $13.7 \pm 1.21$ weeks (range, 12-16 weeks). (Figure 2.) The humeral NSA at final follow-up was $126.4 \pm 14.23$. The mean UCLA score was $22.4 \pm 6.49$ points and was excellent in 2 patients, good in 5 , and poor in 12 . Significant differences of $129.2 \pm 19.40^{\circ}, 112.3 \pm 14.75^{\circ}$ and $44.218 .50^{\circ}$ were found in the mean forward elevation, abduction, and external rotation, as compared with the opposite arm. Significant differences were also noted in the internal rotation of L2-L3(S-T12), as compared with the opposite arm (Table 2).

\section{Comparison of outcomes associated with medial comminuted fracture}

Seven patients had a medial comminuted fracture. Their mean age was $82.7 \pm 4.02 y e a r s$ and mean bone density was $-4.1 \pm 1.34$. The preoperative NSA was $108.2^{\circ} \pm 26.46^{\circ}$, postoperative NSA was $130.8^{\circ}$, and NSA at final follow-up was $111.4^{\circ} \pm 13.45^{\circ}$. Twelve patients had no medial comminuted fracture. Their mean age was $82.0 \pm 2.55$ years and mean bone density was $-3.7 \pm 0.39$. The NSA was $128.8^{\circ} \pm 9.35$ before operation, $135.8^{\circ} \pm 2.03^{\circ}$ just after operation, and $135.1^{\circ} \pm 2.51^{\circ}$ at final follow-up. We found a statistically significant difference between the preoperative NSA and the NSA at final follow-up. In the group with medial comminuted fractures, the mean range of motion was $108.5^{\circ} \pm 10.69^{\circ}$ for forward elevation, $99.3^{\circ}$ $\pm 6.07^{\circ}$ for abduction, and $26.4^{\circ} \pm 12.48^{\circ}$ for the internal rotation of $L 2$. In the group without medial comminuted fracture, statistically significant differences were noted in forward elevation of $139.4^{\circ}$ $\pm 11.64^{\circ}$, external rotation of $120.1^{\circ} \pm 12.79^{\circ}$, internal rotation of $54.5^{\circ} \pm 12.51^{\circ}$, and internal rotation of L4 (S-T12). The UCLA shoulder score showed significant mean differences of $18.3 \pm 1.71$ and $24.7 \pm 5.63$ in the groups with and without medial comminuted fracture, respectively (Table 3 ).

\section{Comparison of outcomes associated with malreduction of tuberosity}

Five patients had malreduction of tuberosity after surgery. Their mean age was $82.6 \pm 2.30$ years and mean bone density was $-3.8 \pm 1.17$. Their preoperative NSA was $120.8^{\circ} \pm 22.92$, postoperative NSA was $134.0^{\circ} \pm 2.23^{\circ}$, and NSA at final follow-up was $122.0^{\circ} \pm 16.04^{\circ}$. Fourteen patients had an anatomical reduction. Their mean age was $82.1 \pm 3.39$ years and mean bone density was $-3.8 \pm 0.77$. Their preoperative NSA was $121.4^{\circ} \pm 19.46^{\circ}$; NSA just after the operation, $134.0^{\circ} \pm 6.30^{\circ}$; and NSA at final followup, $128.0^{\circ} \pm 13.82^{\circ}$. No statistically significant difference was found between the preoperative NSA and NSA at final follow-up. In addition, the clinical results were not satisfactory in the patients with malreduction. However, no statistically significant difference was attained in both groups (Table 4).

\section{Comparison of outcomes according to fracture type}

Nine patients had two-part fractures. Their mean age was $81.1 \pm 2.47 y$ ears and mean bone density was $-3.8 \pm 0.58$. Their preoperative NSA was $116.2^{\circ} \pm 22.96$; postoperative NSA, $134.7^{\circ} \pm 5.89$; and NSA at final follow-up, $127.6^{\circ} \pm 15.94$. Ten patients had three- or four-part fractures. Their mean age was $83.3 \pm 3.33$ years and mean bone density was $-3.8 \pm 1.08$. Their preoperative NSA was $125.8^{\circ} \pm 13.27$, NSA just after the operation was $133.3^{\circ} \pm 5.25$, and NSA at final follow-up was $125.3^{\circ} \pm 13.27$. A statistically significant 
difference was found between the preoperative NSA and the NSA at final follow-up. In the group with twopart fractures, the mean range of motion was $132.2^{\circ} \pm 21.08$ for forward elevation, $115.5^{\circ} \pm 15.89$ for abduction, and $48.8^{\circ} \pm 17.46$ for external and internal rotations to L2(S-T11). In the group with three- or four-part fractures, no statistically significant differences were observed in the forward elevation of $126.0^{\circ} \pm 18.37$, abduction of $109.5^{\circ} \pm 13.83$, external rotation of $40.0^{\circ} \pm 19.29$, and an internal rotation L2/L3(S-T12). The mean UCLA scores of $24.5 \pm 6.20$ and $20.4 \pm 6.39$ for the group with two-part fractures and the group with three- or four-part fractures showed no significant difference (Table 5).

Complications were osteonecrosis in 1 patient and screw penetration in 2 patients. One patient with osteonecrosis was only observed because the pain was not severe. Penetrating screw removal was performed in 1 patient. Other patient did not want to remove the screw, So We decided to conservation for that because it did not affect joint destruction.

\section{Discussion}

Although various methods such as percutaneous K-wire fixation, plate fixation, and arthroplasty have been proposed for the treatment of proximal humeral fracture with osteoporosis, a definite treatment method has not yet been established [23]. In the case of complications such as infection and loss of reduction due to weakening of the fixation, pin fixation should be used when bone quality is good and in the absence of a comminuted fracture [9]. According to Boudard [4], the study did not show that locking plates resulted in better functional outcome, radiographic results or reduction of postoperative complications than intramedullary nail. However, many other literatures have reported various complications for intramedullary nail surgery. It causes various complications such as distraction, nonunion, screw back-out, and rotator cuff tear.[3, 7, 27] In the case of arthroplasty, the incidence rate of complications such as dislocation, nerve damage, and infection ranges from $5 \%$ to $40 \%$ [31]. Furthermore, Patients older than 70 years and patients with chronic debilitating disease showed significantly poorer clinical outcome after hemiarthroplasty for proximal humeral fracture.[22]

Reverse total shoulder arthroplasty for complex proximal humeral fractures is still controversial but RSA has been increasingly used as an alternative to hemiarthroplasty in the recent years.[29] However, RSA cause unique complications, including scapular notching, acromial fracture, and a higher incidence of implant instability. We need to long-term outcome studies to define RSA prosthesis longevity and possible late implant failure in elderly patients for proximal humeral fractures.[25, 29]

When we cannot achieve adequate reduction due to comminuted fracture at the surgical neck including medial calcar and severe bone loss, we can't expect a medial buttress even after reduction. In that cases, varus deformity and loss of fracture were reported. The reason for this is that medial comminuted fracture is associated with blood flow injury, poor bone quality, and high energy damage [19]. The authors also reported that varus deformity was more severe in the medial comminuted fractures. After operation, we achieved restoration of the NSA but found no statistically significant difference at final follow-up. Clinical results also showed decreases in the range of motion and clinical performance. For this reason, 
Gardner et al. [12] proposed a fibular strut allograft augmentation and reported good results when the medial support was difficult.

It is often accompanied by shoulder stiffness and scapular dyskinesia after proximal humeral fracture. Thus, various rehabilitation methods have been introduced to prevent this [12, 28]. Aggressive rehabilitation can reduce the many discomforts associated with stiffness. The authors presented various exercise methods to patients, including scapular, stretching, and strengthening exercises. However, in elderly patients, compliance was poor and aggressive rehabilitation was difficult because of pain, and these factors are also considered to contribute to the poor clinical outcome.

Open plate fixation was generally good, but complication and reoperation rates of $15 \%$ and $12.7 \%$ were reported, respectively [11]. Various complications such as malunion, loss of fracture reduction, metal failure, nonunion, and osteonecrosis have been reported. In $8 \%$ of patients, screw penetration was the most common cause of reoperation [18]. Two patients had screw penetration, one of whom had reoperation to remove the screw. To prevent this, during the operation, the length of the screw should examined using $\mathrm{C}$-arm imaging. Furthermore, the far-cortex should be examined with a depth gage, based on which the screw length can be decided.

Furthermore, we need to understand the screw configurations according to the plate type and proceed with the surgery. Of course, preoperatively we have to check evaluation of bone quality of the patient, accurate analysis of the fracture type, plate selection.

The incidence rate of osteonecrosis after surgical treatment of proximal humeral fractures is reported to be $3-35 \%$ [26]. Especially in the proximal humeral head, after 48 hours, the frequency of this is high. In these patients, the frequency of shifting to arthroplasty was high.(80\%) The authors also performed surgical treatment in 1 patient on postoperative day 4, which resulted in osteonecrosis. However, considering the age and activity of this patient, we decided to perform conservative treatment.

Recently, the number of reverse total shoulder arthroplasty cases has been increasing among complex humeral fractures. The results are excellent as compared with those of hemiarthroplasty, and the 5- and 10-year survival rates are $94 \%$ and $91 \%$, respectively. However, the technique of reverse total shoulder arthroplasty for fractures was found to be more difficult than the procedure for rotator cuff disease, and the functional score decreased from 6 years after the procedure, so that the Constant-Murley score was maintained at $\geq 30$ points in $60 \%$ of patients only [14]. Generally, the complication rate of reverse total shoulder arthroplasty is reported to be $19-68 \%$. Furthermore, complication rate of reverse total shoulder arthroplasty for fracture vary among studies and range from $5 \%$ to $40 \%$ such as periprothetic fracture, infection, dislocation and nerve paly.[14] Cheung et al reported that treatment of the these complications is difficult.[6] Therefore, reverse total shoulder arthroplasty is not an easy choice for treating proximal humeral fractures even in the elderly [10].

One of the study's limitations is that, as a retrospective study, the findings may have been influenced by selection bias. Although we considered Neer classification and medial comminution, we did not make an 
analysis of the severity of fractures exactly. Second, the total number of cases are only 19 , therefore it is difficult to anticipate a statistically significant difference. Third, because the study design consisted of a relatively short follow-up, long-term complication, such as osteonecrosis, arthritis, cannot be out-ruled. Lastly, It has the disadvantage of not comparing with other surgical methods.

\section{Conclusions}

In the surgical treatment of proximal humeral fractures in patients aged $>80$ years, use of a locking plate attained bone union with relatively satisfactory results. However, postoperative shoulder motion was significantly reduced compared to normal. Therefore, it seems that active physical therapy is needed. Other surgical methods should be considered for patients with complex displaced fractures, especially those with medial comminuted fractures.

\section{Abbreviations}

UCLA score(University of California, Los Angeles, shoulder score), NSA (neck-shaft angle), CT (computed tomography)

\section{Declarations}

This was a retrospective study, and final approval of informed consent exemption by the institutional review board was obtained (IRB NO. 05-2019-013).

Consent to publish: We obtained consent for publication

Availability of data and materials: The datasets used and/or analyzed during the current study

are available from the corresponding author on reasonable request.

Competing interests: The authors declare that they have no competing interests

Funding: No funding was obtained for this study

Authors' Contributions: all authors have read and approved the manuscript

WCS: $1^{\text {st }}$ author, data analysis

SWK: Corresponding author, data analysis

SMS: Statistical support

HB: Data analysis

Acknowledgements: Not Applicable 


\section{References}

1. Amstutz HC, Sew Hoy AL, Clarke IC. UCLA anatomic total shoulder arthroplasty. Clin Orthop Relat Res. 1981:7-20.

2. Axelsson $\mathrm{KF}$, Wallander $\mathrm{M}$, Johansson $\mathrm{H}$, Lundh $\mathrm{D}$, Lorentzon $\mathrm{M}$. Hip fracture risk and safety with alendronate treatment in the oldest-old. J. Intern. Med. 2017;282:546-559.

3. Boileau P, Pennington SD, Alami G. Proximal humeral fractures in younger patients: fixation techniques and arthroplasty. J Shoulder Elbow Surg. 2011;20:S47-60.

4. Boudard G, Pomares G, Milin L, Lemonnier I, Coudane H, Mainard D, Delagoutte JP. Locking plate fixation versus antegrade nailing of 3- and 4-part proximal humerus fractures in patients without osteoporosis. Comparative retrospective study of 63 cases. Orthop. Traumatol. Surg. Res. 2014;100:917-924.

5. Calvo E, de Miguel I, de la Cruz JJ, Lopez-Martin N. Percutaneous fixation of displaced proximal humeral fractures: indications based on the correlation between clinical and radiographic results. $J$ Shoulder Elbow Surg. 2007;16:774-781.

6. Cheung E, Willis M, Walker M, Clark R, Frankle MA. Complications in reverse total shoulder arthroplasty. J Am Acad Orthop Surg. 2011;19:439-449.

7. Congia S, Palmas A, Marongiu G, Capone A. Is antegrade nailing a proper option in 2- and 3-part proximal humeral fractures? Musculoskelet. Surg. 2019.

8. dos Santos VR, Gobbo LA, Christofaro DG, Gomes IC, Mota J, Gobbi S, Freitas Junior IF. Osteoarticular diseases and physical performance of Brazilians over 80 years old. Cien Saude Colet. 2016;21:423-430.

9. El-Alfy BS. Results of the percutaneous pinning of proximal humerus fractures with a modified palm tree technique. Int Orthop. 2011;35:1343-1347.

10. Familiari F, Rojas J, Nedim Doral M, Huri G, McFarland EG. Reverse total shoulder arthroplasty. EFORT Open Rev. 2018;3:58-69.

11. Gupta AK, Harris JD, Erickson BJ, Abrams GD, Bruce B, McCormick F, Nicholson GP, Romeo AA. Surgical management of complex proximal humerus fractures-a systematic review of 92 studies including 4500 patients. J Orthop Trauma. 2015;29:54-59.

12. Hodgson S. Proximal humerus fracture rehabilitation. Clin Orthop Relat Res. 2006;442:131-138.

13. Jo YH, Lee KH, Lee BG. Surgical trends in elderly patients with proximal humeral fractures in South Korea: a population-based study. BMC Musculoskelet Disord. 2019;20:136.

14. Jobin CM, Galdi B, Anakwenze OA, Ahmad CS, Levine WN. Reverse shoulder arthroplasty for the management of proximal humerus fractures. J Am Acad Orthop Surg. 2015;23:190-201.

15. Koval KJ, Gallagher MA, Marsicano JG, Cuomo F, McShinawy A, Zuckerman JD. Functional outcome after minimally displaced fractures of the proximal part of the humerus. $J$ Bone Joint Surg Am. 1997;79:203-207. 
16. LaMartina J, 2nd, Christmas KN, Simon P, Streit JJ, Allert JW, Clark J, Otto RJ, Abdelfattah A, Mighell MA, Frankle MA. Difficulty in decision making in the treatment of displaced proximal humerus fractures: the effect of uncertainty on surgical outcomes. J Shoulder Elbow Surg. 2018;27:470-477.

17. Lee CW, Shin SJ. Prognostic factors for unstable proximal humeral fractures treated with lockingplate fixation. J Shoulder Elbow Surg. 2009;18:83-88.

18. McMillan TE, Johnstone AJ. Primary screw perforation or subsequent screw cut-out following proximal humerus fracture fixation using locking plates: a review of causative factors and proposed solutions. Int Orthop. 2018;42:1935-1942.

19. Osterhoff G, Hoch A, Wanner GA, Simmen HP, Werner CM. Calcar comminution as prognostic factor of clinical outcome after locking plate fixation of proximal humeral fractures. Injury. 2012;43:16511656.

20. Owsley KC, Gorczyca JT. Fracture displacement and screw cutout after open reduction and locked plate fixation of proximal humeral fractures [corrected]. J Bone Joint Surg Am. 2008;90:233-240.

21. Palvanen M, Kannus P, Niemi S, Parkkari J. Update in the epidemiology of proximal humeral fractures. Clin Orthop Relat Res. 2006;442:87-92.

22. Park YK, Kim SH, Oh JH. Intermediate-term outcome of hemiarthroplasty for comminuted proximal humerus fractures. J Shoulder Elbow Surg. 2017;26:85-91.

23. Ring D. Current concepts in plate and screw fixation of osteoporotic proximal humerus fractures. Injury. 2007;38 Suppl 3:S59-68.

24. Sabesan VJ, Lombardo D, Petersen-Fitts G, Weisman M, Ramthun K, Whaley J. National trends in proximal humerus fracture treatment patterns. Aging Clin. Exp. Res. 2017;29:1277-1283.

25. Schairer WW, Nwachukwu BU, Lyman S, Craig EV, Gulotta LV. Reverse shoulder arthroplasty versus hemiarthroplasty for treatment of proximal humerus fractures. J Shoulder Elbow Surg. 2015;24:15601566.

26. Schnetzke M, Bockmeyer J, Loew M, Studier-Fischer S, Grutzner PA, Guehring T. Rate of avascular necrosis after fracture dislocations of the proximal humerus: Timing of surgery. Obere Extrem. 2018;13:273-278.

27. Schumaier A, Grawe B. Proximal Humerus Fractures: Evaluation and Management in the Elderly Patient. Geriatr Orthop Surg Rehabil. 2018;9:2151458517750516.

28. Schwickert L, Klenk J, Stahler A, Becker C, Lindemann U. Robotic-assisted rehabilitation of proximal humerus fractures in virtual environments: a pilot study. Z. Gerontol. Geriatr. 2011;44:387-392.

29. Torrens C, Alentorn-Geli E, Mingo F, Gamba C, Santana F. Reverse shoulder arthroplasty for the treatment of acute complex proximal humeral fractures: Influence of greater tuberosity healing on the functional outcomes. J Orthop Surg (Hong Kong). 2018;26:2309499018760132.

30. Yamamoto A, Takagishi K, Osawa T, Yanagawa T, Nakajima D, Shitara H, Kobayashi T. Prevalence and risk factors of a rotator cuff tear in the general population. J Shoulder Elbow Surg. 2010;19:116120. 
31. Zumstein MA, Pinedo M, Old J, Boileau P. Problems, complications, reoperations, and revisions in reverse total shoulder arthroplasty: a systematic review. J Shoulder Elbow Surg. 2011;20:146-157.

\section{Tables}

Table 1. Preoperative demographic data of patients $(\mathrm{N}=19)$

\begin{tabular}{|c|c|}
\hline Variable & Values \\
\hline Number of patients & 19 \\
\hline Mean(range) age, years & $82.2(80-91)$ \\
\hline Mean(range) follow-up duration, months & $15.4(12-24)$ \\
\hline Sex & Male 4, Female 15 \\
\hline Mean (range) bone mineral density & -3.8 (range, $-3.1 \sim-5.9$ ) \\
\hline Cause & Fall 17, Traffic accident 2 \\
\hline \multicolumn{2}{|l|}{ Type of fracture (Neer classification) } \\
\hline 2-parts & 9 \\
\hline 3-parts & 8 \\
\hline 4-parts & 2 \\
\hline
\end{tabular}

Table 2. Shoulder range of motion after final follow-up

\begin{tabular}{llll}
\hline & Fracture side & Opposite side & p-value \\
\hline Forward elevation & $129.2 \pm 19.40^{\circ}$ & $152.8 \pm 12.56^{\circ}$ & 0.02 \\
Abduction & $112.3 \pm 14.75^{\circ}$ & $127.5 \pm 12.36^{\circ}$ & 0.02 \\
\hline External rotation & $44.218 .50^{\circ}$ & $77.5 \pm 7.05^{\circ}$ & 0.05 \\
\hline Internal rotation & L2-L3 & T12-L5 & 0.00 \\
\hline
\end{tabular}


Table 3. Surgical results related to medial comminuted fracture

\begin{tabular}{llll}
\hline & medial comminution & No medial comminution & p-value \\
\hline Number of patients & 7 & 12 & \\
\hline Age, years & $82.7 \pm 4.02$ & $82.0 \pm 2.55$ & 0.592 \\
\hline Follow-up duration & $15.7 \pm 6.36$ & $15.2 \pm 4.49$ & 0.650 \\
BMD & $-4.1 \pm 1.34$ & $-3.7 \pm 0.39$ & 0.592 \\
\hline UCLA score & $18.3 \pm 1.71$ & $24.7 \pm 5.63$ & 0.007 \\
\hline Preoperative NSA, degrees & $108.2 \pm 26.46$ & $128.8 \pm 9.35$ & 0.017 \\
\hline Postoperative NSA, degrees & $130.8 \pm 7.96$ & $135.8 \pm 2.03$ & 0.340 \\
\hline Last follow-up NSA, degrees & $111.4 \pm 13.45$ & $135.1 \pm 2.51$ & 0.000 \\
\hline Forward elevation, degrees & $108.5 \pm 10.69$ & $139.4 \pm 11.64$ & 0.000 \\
\hline Abduction, degrees & $99.3 \pm 6.07$ & $120.1 \pm 12.79$ & 0.000 \\
\hline External rotation, degrees & $26.4 \pm 12.48$ & $54.5 \pm 12.51$ & 0.000 \\
\hline Internal rotation, degrees & L2 & L4 & 0.479 \\
\hline
\end{tabular}

Data are presented as mean $\pm \mathrm{SD}$.

Table 4. Surgical results related malreduction of tuberosity

\begin{tabular}{llll}
\hline & malreduction & Anatomical reduction & p-value \\
\hline Number of patients & 5 & 14 & \\
\hline Age, years & $82.6 \pm 2.30$ & $82.1 \pm 3.39$ & 0.687 \\
\hline Follow-up duration & $17.2 \pm 7.56$ & $14.8 \pm 4.07$ & 0.687 \\
\hline BMD & $-3.8 \pm 1.17$ & $-3.8 \pm 0.77$ & 0.500 \\
\hline UCLA score & $19.6 \pm 4.82$ & $23.3 \pm 6.86$ & 0.343 \\
\hline Preoperative NSA, degrees & $120.8 \pm 22.92$ & $121.4 \pm 19.46$ & 0.893 \\
\hline Postoperative NSA, degrees & $134.0 \pm 2.23$ & $134.0 \pm 6.30$ & 0.444 \\
Last follow-up NSA, degrees & $122.0 \pm 16.04$ & $128.0 \pm 13.82$ & 0.500 \\
\hline Forward elevation, degrees & $124.0 \pm 16.73$ & $130.7 \pm 20.55$ & 0.070 \\
\hline Abduction, degrees & $101.4 \pm 8.36$ & $116.0 \pm 14.95$ & 0.219 \\
\hline External rotation, degrees & $35.0 \pm 18.70$ & $47.5 \pm 17.94$ & 1.000 \\
\hline Internal rotation, degrees & L2-L3 & L2-L3 & 1.000 \\
\hline
\end{tabular}

Data are presented as mean $\pm \mathrm{SD}$. 
Table 5. Surgical results related to fracture type (Neer classification)

\begin{tabular}{llll}
\hline & 2-part & 3,4 -part & p-value \\
\hline Number of patients & 9 & 10 & \\
\hline Age, years & $81.1 \pm 2.47$ & $83.3 \pm 3.33$ & 0.211 \\
\hline Follow-up duration & $16.1 \pm 4.59$ & $14.8 \pm 5.67$ & 0.243 \\
BMD & $-3.8 \pm 0.58$ & $-3.8 \pm 1.08$ & 0.447 \\
\hline UCLA score & $24.5 \pm 6.20$ & $20.4 \pm 6.39$ & 0.211 \\
\hline Preoperative NSA, degrees & $116.2 \pm 22.96$ & $125.8 \pm 16.24$ & 0.661 \\
Postoperative NSA, degrees & $134.7 \pm 5.89$ & $133.3 \pm 5.25$ & 0.095 \\
\hline Last follow-up NSA, degrees & $127.6 \pm 15.94$ & $125.3 \pm 13.27$ & 0.356 \\
\hline Forward elevation, degrees & $132.2 \pm 21.08$ & $126.0 \pm 18.37$ & 0.400 \\
\hline Abduction, degrees & $115.5 \pm 15.89$ & $109.5 \pm 13.83$ & 0.447 \\
\hline External rotation, degrees & $48.8 \pm 17.46$ & $40.0 \pm 19.29$ & 0.447 \\
\hline Internal rotation, degrees & L2 & L2-L3 & 0.356 \\
\hline
\end{tabular}

Data are presented as mean $\pm \mathrm{SD}$.

\section{Figures}




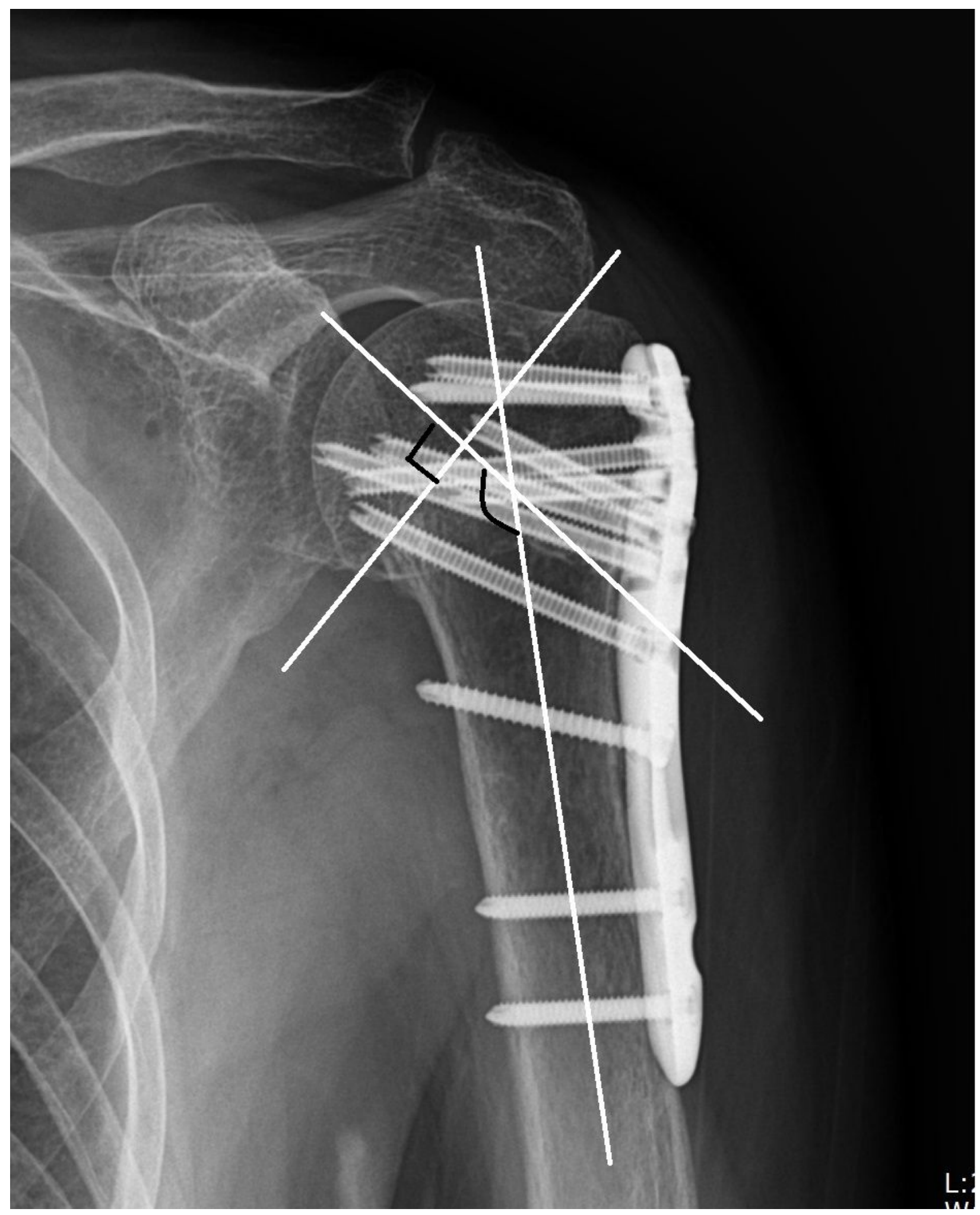

\section{Figure 1}

Neck-shaft angle: the angle formed between the line perpendicular to the articular surface and the line following the humeral shaft. 

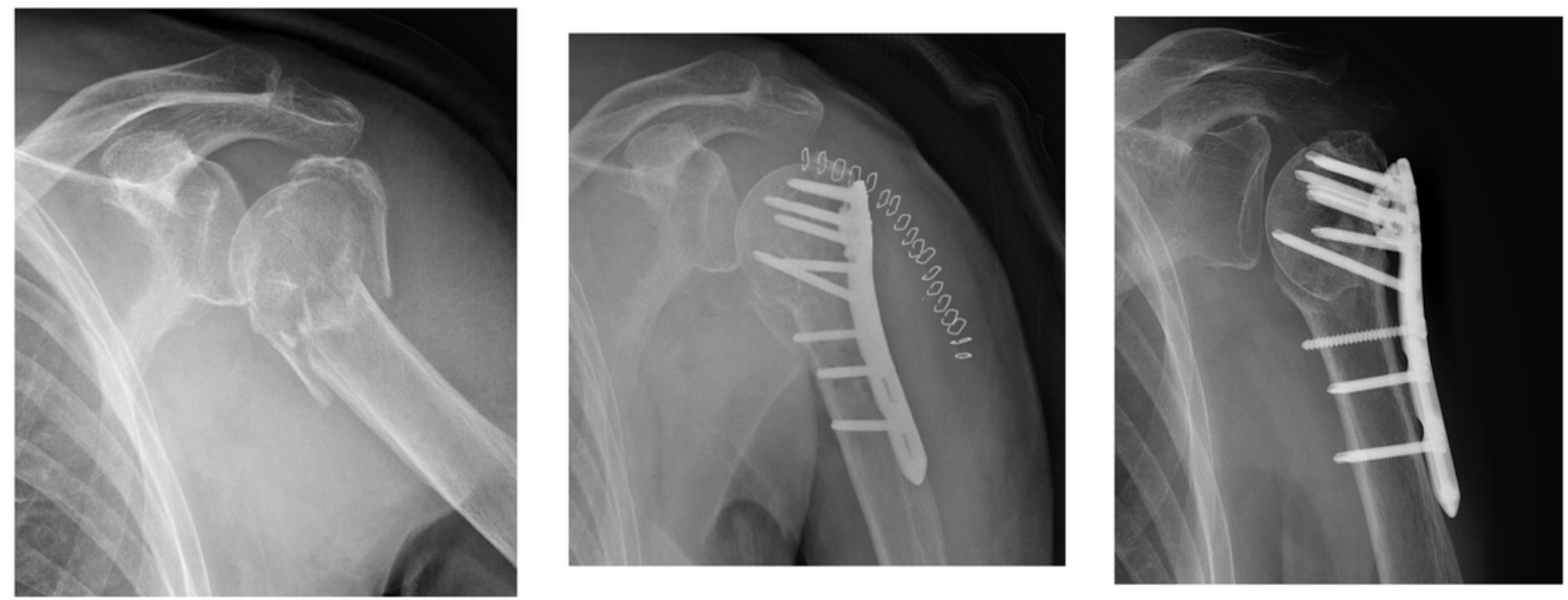

Figure 2

A-C. Radiography of the Left shoulder in a 85-year-old patient A. Preoperative anteroposterior radiograph showing medial comminuted fracture B. Postoperative anteroposterior radiograph C. Postoperative anteroposterior radiograph showing varus deformity at the 12-month follow-up 